\title{
I ntroducción a la estimación de la carga de los factores de riesgo en Cuba
}

\section{I ntroduction to the estimation of the burden of risk factors in Cuba}

\author{
Armando H. Seuc Jo'; Emma Domínguez Alonsol' \\ 'Investigador Titular. Instituto de Angiología y Cirugía Vascular. La Habana, Cuba. \\ "Especialista de II Grado en Bioestadística. Instituto Nacional de Endocrinología. La \\ Habana, Cuba.
}

\section{RESUMEN}

En Cuba varios estudios han estimado la carga de distintas enfermedades y lesiones, empleando como indicador de carga los Años de Vida Potencial Perdidos por Muertes Prematuras, entre otros. La utilidad de estos estudios se debe, especialmente, a su vinculación estrecha con las afectaciones que distintas enfermedades y lesiones provocan en la esperanza de vida. Sin embargo, la estimación de la carga asociada a determinados factores de riesgo es al menos tan importante, sobre todo desde el muy relevante enfoque preventivo y de promoción de salud. En este trabajo se revisan los conceptos básicos vinculados a estos estudios, los pasos fundamentales que requiere su ejecución, y se reflexiona sobre cómo adaptar e implementar estos pasos generales al contexto nacional.

Palabras clave: Estudio de Carga de Factores de Riesgo, Años de Vida Potencial Perdidos por Muertes Prematuras, esperanza de vida, factores de riesgo, Cuba.

\section{ABSTRACT}


Several studies in Cuba have estimated the burden of several diseases and lesions by using the Potential Years of Life Lost by Premature Death as burden indicator, among others. The use of these studies is mainly due to their close linking with the effects of various diseases and lesions on the life expectancy. However, the estimation of the burden associated to certain risk factors is also important, particularly from the preventive and health promotion viewpoints. This paper reviewed the basic concepts associated to these studies, the fundamental steps for their implementation, and made reflections on how these general steps may be adapted to and implemented in our national setting.

Key words: Burden of risk factors study, Potential Years of Life Lost by Premature Death, life expectancy, risk factors, Cuba.

\section{NTRODUCCI ÓN}

El tema de la estimación de la carga de las enfermedades atribuible a determinados factores de riesgo es de gran actualidad. ${ }^{1-3}$ En sus estudios globales de carga de enfermedades para los años 1990 y 2000, ${ }^{4-6}$ la Organización Mundial de la Salud (OMS) incluyó módulos para estimar la carga de factores de riesgo.

Estos estudios tienen también gran relevancia para Cuba donde la medicina preventiva está priorizada. Este trabajo presenta un resumen de los principales conceptos y métodos asociados con la estimación de la carga de los factores de riesgo, de manera que los lectores se vayan familiarizando con estos temas. Al mismo tiempo, reflexionar acerca de cómo implementar esta tecnología en el país, tomando en cuenta sus condiciones específicas, recursos informativos disponibles, y otros.

El trabajo se divide en dos secciones principales. En la primera, se introducen algunos conceptos y métodos generales y esenciales para este tipo de estudios a través de la descripción de los principales pasos que se requieren para ejecutarlos. En la segunda sección, se sugiere algunas adaptaciones y/o elecciones particulares que parecen adecuadas al caso cubano, tomando en cuenta la experiencia acumulada en el cálculo de la Carga de Enfermedades en el país.

\section{CONCEPTOS Y MÉTODOS GENERALES}

Los estudios de carga de factores de riesgo, o evaluaciones comparativas de riesgos (términos que se usaran indistintamente), tienen como objetivo evaluar de manera sistemática los cambios que se producen en el nivel de salud de una población debido a la reducción del nivel de exposición a un factor de riesgo, en comparación con reducciones "similares" en otros factores de riesgo.

Aunque indiscutiblemente relacionados, es conveniente precisar que los estudios de Carga de Factores de Riesgo no son estudios de análisis de intervenciones. En estos últimos se analiza el impacto de una determinada acción o grupo de acciones sobre 
el nivel de salud en una población. Por otro lado, la reducción en la exposición al factor de riesgo que se asume en un estudio de Carga de Factores de Riesgo no necesariamente responde a una determinada acción; incluso, puede que esa reducción no sea factible o probable en un determinado contexto de lugar y momento.

Para el propósito de mejorar de manera creciente el estado de salud de una población, el conocimiento del "aporte" de distintos factores de riesgo sobre el nivel de salud (usualmente sobre su falta) en una población (expresada generalmente en términos de mortalidad, muerte prematura, discapacidad, o DALYs (Años de Vida Ajustados por Discapacidad, AVAD), es al menos tan importante como conocer el "aporte" de distintas enfermedades y lesiones.

En el último caso, la estimación de la carga de las enfermedades, la información obtenida es especialmente relevante para orientar las acciones relativas a los servicios de salud (tratamiento y/o rehabilitación); en el primer caso, la estimación de la carga de los factores de riesgo, la información obtenida es especialmente útil para orientar las acciones relativas a la prevención y promoción de salud.

El enfoque clásico y estándar en epidemiología para evaluar el impacto sobre la (falta de) salud de un factor de riesgo es a través de la Fracción Atribuible (FA), dada por la expresión:

$$
F A=\frac{P(R R-1)}{P(R R-1)+1}
$$

donde $\mathrm{P}$ es la prevalencia de la exposición al factor de riesgo, y RR es el riego relativo con respecto a la "no exposición." Por otro lado, si en vez de estar la población expuesta a un nivel específico de exposición al factor de riesgo, lo que se tiene es una distribución de exposición de la población al factor de riesgo, la que se compara con una distribución de exposición hipotética "de referencia", entonces [1] se convierte en [2]:

$$
F A=\frac{\sum_{i=1}^{n} P_{i}\left(R R_{i}-1\right)-\sum_{i=1}^{n} P_{i}(R R-1)}{\sum_{i=1}^{n} P_{i}\left(R R_{i}-1\right)}
$$

donde $\mathrm{n}$ es el número de categorías de exposición, $\mathrm{P}_{\mathrm{i}}$ la proporción de la población en la i-ésima categoría de exposición, $\mathrm{P}_{\mathrm{i}}{ }_{\mathrm{i}}$ la proporción de la población en la i-ésima categoría de exposición en la distribución hipotética de referencia, y $R_{i}$ el riesgo relativo asociado a la i-ésima categoría de exposición.

Si se asume que en la distribución hipotética de referencia $\mathrm{P}_{\mathrm{i}}$ el riesgo relativo $R R_{i}=1$ entonces [2] se convierte en [3]: 


$$
F A=\frac{\sum_{i=1}^{n} P_{i}\left(R R_{i}-1\right)-1}{\sum_{i=1}^{n} P_{i}\left(R R_{i}-1\right)}
$$

En los estudios de carga de factores de riesgo la estimación de las fracciones atribuibles juega un rol central. La Fracción Atribuible cuantifica la reducción proporcional que se produce en la "carga negativa" cuando la exposición al factor de riesgo se reduce de $\mathrm{P}_{\mathrm{i}}$ a $\mathrm{P}^{\prime}{ }_{\mathrm{i}}$.

Para estimar la fracción de la carga negativa de la enfermedad $\mathrm{j}$ atribuible al factor de riesgo i, donde la carga negativa puede expresarse en términos de mortalidad (M), mortalidad prematura (YLL, según sus siglas en inglés), discapacidad (YLD, según sus siglas en inglés), o DALYs (suma de los YLL y los YLD), se usa entonces la expresión [3] de la Fracción Atribuible correspondiente a la pareja "factor de riesgo-enfermedad" objeto de estudio, como se presenta en las siguientes fórmulas:

Mortalidad Atribuible:

YLL Atribuible:

YLD Atribuible:

$A M i j=F A(M) i j * M j$

$A Y L L i j=F A(Y L L) i j * Y L L j$

AYLD ij $=F A(Y L D) i j * Y L D j$

Carga Total (DALYs) Atribuible:

ADALYs $i j=A Y L L i j+A Y L D i j$

Nota: la Fracción Atribuible (FA) puede en general variar según el indicador de carga negativa empleado.

\section{Principales pasos para realizar un estudio de Carga de Factores de Riesgo}

Llegado este punto, se puede ahora presentar los principales pasos para la ejecución de un estudio de Carga de Factores de Riesgo con ligera modificación de los presentados en otros estudios. ${ }^{7}$

1. Elección de los factores de riesgo.

2. Elección de las enfermedades y lesiones relevantes que tienen como causa cada uno de los factores de riesgo en 1.

3. Elección de la variable de exposición adecuada para cada factor de riesgo en 1.

4. Obtención de información acerca de la distribución de exposición en la población a cada uno de los factores de riesgo en 1 .

5. Especificación de la relación (riesgo relativo) entre cada enfermedad en 2 y los relevantes factores de riesgo en 1 . Para ello se deberá hacer una revisión exhaustiva de la literatura.

6. Elección de la distribución de exposición hipotética de referencia (o escenario alternativo de referencia) para cada uno de los factores de riesgo en 1.

7. Cálculo de la carga (YLL, YLD, DALYs, y otros) para cada enfermedad en 2. 
8. Cálculo de la carga por cada enfermedad en 2 atribuible a los relevantes factores de riesgo en 1, usando las fórmulas [3] y [4].

9. Análisis de la confiabilidad de los resultados obtenidos.

A continuación se describe brevemente las acciones y análisis comprendidos en cada uno de estos pasos.

1. Elección de los factores de riesgo.

Esta elección debe hacerse a partir de una serie de criterios tales como : i) las prioridades del lugar donde se hace el estudio, ii) la evidencia existente sobre ese factor, su impacto, y su relación causal con determinadas enfermedades, iii) la posibilidad de intervenir para reducir el grado de exposición de la población a ese factor, entre otros. En sus estudios de carga de factores de riesgo, la OMS ha trabajado usualmente con 20 factores de riesgo (recuadro 1 ).

2. Elección de las enfermedades y lesiones relevantes que tienen como causa cada uno de los factores de riesgo en 1 .

Debe existir una evidencia científica importante que fundamente la relación causal entre los factores de riesgo en 1 y las enfermedades asociadas correspondientes. Existen distintos criterios para establecer causalidad en casos específicos, pero una referencia válida todavía insoslayable es la presentada por Sir Bradford Hill en $1965 .^{8}$

Por otro lado, la elección de las enfermedades y lesiones a incluir en el estudio deberá tomar en cuenta si su carga ha sido estimada recientemente en la región o país de que se trate. La OMS ha empleado en sus estudios de Carga Global una relación de más de 100 enfermedades y lesiones, relación demasiado amplia para ser reproducida en este artículo.

Existen procedimientos ${ }^{1,3}$ que permiten aglutinar la carga atribuible a un factor de riesgo con respecto a un grupo de enfermedades (p.ej. "tumores malignos") a partir de la carga de ese factor con respecto a cada una de las enfermedades que conforman ese grupo (en el ejemplo, los tumores específicos). Estos procedimientos ${ }^{6}$ permitirían estimar las cargas atribuibles de los factores de riesgo incluidos en el estudio con respecto a los 21 grupos de enfermedades que se reproducen en el recuadro 2 .

Recuadro 1. Factores de riesgo usualmente empleados por la OMS en sus estudios de carga de factores de riesgo 
1. Bajo peso infantil y materno (Childhood and maternal underweight).

2. Sexo no seguro (Unsafe sex).

3. Hipertensión (High blood pressure).

4. Tabaco (Smoking and oral tobacco use).

5. Ingestión de alcohol (Alcohol use).

6. Agua, saneamiento e higiene inadecuados (Unsafe water, sanitation and hygiene).

7. Colesterol elevado (High cholesterol).

8. Contaminación del aire en viviendas por uso de combustible sólido (Indoor air pollution from household use of solid fuels).

9. Anemia por deficiencia de hierro (Iron deficiency anaemia). 10. Sobrepeso y obesidad [Overweight and obesity (high BMI )].

11. Deficiencia de Zinc (Zinc deficiency).

12. Consumo de frutas y vegetales insuficiente (Low fruit and vegetable consumption).

13. Deficiencia de vitamina A (vitamin A deficiency).

14. Sedentarismo (Physical inactivity).

15. Exposición a plomo (Lead exposure).

16. Drogadicción (Illicit drug use).

17. Factores de riesgo ocupacionales (Occupational risk factors for injury).

18. Jeringas contaminadas en centros de salud (Contaminated injections in health care settings).

19. No uso o uso de inadecuados métodos contraceptivos (Non-use and use of ineffective methods of contraception). 20. Abuso sexual infantil (Child sexual abuse).

Recuadro 2. Relación de categorías de enfermedades empleada en varios estudios de carga global de enfermedades de la OMS

\author{
1. Enfermedades infecciosas y \\ parasitarias. \\ 2. Infecciones respiratorias. \\ 3. Condiciones maternas. \\ 4. Condiciones que surgen en \\ el periodo perinatal \\ 5. Deficiencias nutricionales. \\ 6. Tumores malignos. \\ 7. Otros tumores. \\ 8. Diabetes mellitus. \\ 9. Alteraciones endocrinas. \\ 10. Condiciones \\ neuropsiquiátricas. \\ 11. Enfermedades de los \\ órganos de los sentidos. \\ 12. Enfermedades \\ cardiovasculares. \\ 13. Enfermedades \\ respiratorias. \\ 14. Enfermedades digestivas. \\ 15. Enfermedades \\ genitourinarias.
}




\author{
16. Enfermedades de la piel. \\ 17. Enfermedades musculo- \\ esqueléticas. \\ 18. Anomalías congénitas. \\ 19. Condiciones orales. \\ 20. Lesiones no intencionales. \\ 21. Lesiones intencionales.
}

En los estudios de carga de enfermedades en Cuba se ha empleado otro atractivo listado de 20 (grupos de) enfermedades, ${ }^{9}$ para las cuales, hasta donde se conoce, no existe evidencia sólida ni pertinente acerca de su relación (riesgos relativos) con los correspondientes factores de riesgo.

3. Elección de la variable de exposición adecuada para cada factor de riesgo en el paso 1.

La elección de los factores de riesgo a estudiar (paso 1) conlleva definir las correspondientes variables de exposición y sus unidades de medida. Por ejemplo, si el factor de riesgo es "ingestión de alcohol", hay que decidir si la variable de exposición es "cantidad de tragos" diarios (o semanales), o "peso en gramos de alcohol ingerido" diariamente (semanalmente). Habrá que decidir también cuáles factores de riesgo se medirán usando categorías discretas, y cuáles se medirán en una escala continua.

La decisión acerca de cuál variable de exposición usar para cada factor de riesgo se debe decidir en última instancia según la cantidad y calidad de la evidencia acerca de la influencia del correspondiente factor de riesgo. Por ejemplo, si está demostrado que la influencia del "uso de tabaco" sobre cáncer de pulmón se basa en la frecuencia e intensidad del hábito de fumar, usar como variable de exposición la prevalencia de fumadores no resuelve realmente el problema.

Las categorías a usar en cada variable de exposición deben adaptarse lo más posible a las categorías empleadas en los estudios que han evaluado con calidad y rigor los riesgos relativos del correspondiente factor de riesgo.

4. Obtención de información acerca de la distribución de exposición en la población a cada uno de los factores de riesgo en 1.

Una vez decididos los factores de riesgo (paso 1) y las correspondientes variables de exposición (paso 3), se requiere conocer el grado de exposición de la población de interés a estos factores a través de las variables de exposición decididas. Este grado de exposición deberá desglosarse según sexo y (grupos de) edad; la OMS ha usado en sus estudios de carga de factores de riesgo, ocho grupos de edad: 0-4, 5$14,15-29,30-44,45-59,60-69,70-79$ y 80 y + años.

Dos de las principales fuentes para obtener esta información son: i) la revisión de la literatura, ii) encuestas sobre prevalencia de factores de riesgo, ad hoc o periódicas. La segunda fuente es en general más confiable, pero también más costosa.

Otras valiosas fuentes sobre el grado de exposición pueden ser expertos de instituciones gubernamentales y académicas, y datos de producción y/o comercialización de productos relacionados con la definición de los factores de riesgo estudiados. 
5. Especificación de la relación (riesgo relativo) entre cada enfermedad en 2 y los relevantes factores de riesgo en 1 .

La especificación de los riesgos relativos para cada pareja "factor de riesgoenfermedad" objeto de interés es uno de los pasos fundamentales para obtener las necesarias Fracciones Atribuibles ya discutidas previamente.

Aunque en general el valor de los riesgos relativos se mantiene relativamente estable de una (sub) población a otra, es posible que en algunos casos no sea así por lo que hay que estar alerta ante una situación como esta.

A partir de una revisión exhaustiva de la literatura la OMS ha estimado estos factores de riesgo para una lista de aproximadamente 20 factores de riesgo y una amplia relación de enfermedades y lesiones causadas por estos factores. En una primera aproximación y a falta de evidencia alternativa sólida, estas estimaciones deberán usarse para los cálculos.

6. Elección de la distribución de exposición hipotética de referencia (o escenario alternativo de referencia) para cada uno de los factores de riesgo en 1.

Para cada uno de los 20 factores de riesgo en el recuadro 1, la OMS ha estipulado una distribución mínimo-teórica de referencia, con respecto a la cual se han estimado las cargas atribuibles para cada pareja "factor de riesgo-enfermedad." Otros escenarios alternativos "de transición" hacia este mínimo-teórico han sido sugeridos y recomendados. Concretamente, reducciones del 10, 20, o $30 \%$, en el nivel de exposición con respecto a la reducción implícita en la exposición mínimoteórica se han recomendado como escenarios útiles debido a que son reducciones, en la mayoría de los casos, probables.

La distribución de exposición "mínimo-teórica" se ha definido como aquella que genera el menor riesgo posible (por ejemplo, cero consumo de tabaco). En el caso de exposiciones "fisiológicas", como la tensión arterial sistólica, la determinación del mínimo-teórico es más compleja; para este caso particular la OMS ha definido el mínimo-teórico como la distribución de exposición con media de $115 \mathrm{mmHg}$ y desviación estándar $6 \mathrm{mmHg}$.

\section{Cálculo de la carga (YLL, YLD, DALYs, y otros) para cada enfermedad en 2.}

La carga negativa de las enfermedades (seleccionadas en el paso 2), a distribuir entre los factores de riesgo pertinentes (seleccionados en el paso 1), puede cuantificarse de distintas maneras. La más fácil y frecuente es en términos de mortalidad; la más completa es los DALYs.

Para el cálculo de los YLL, YLD y los DALYs, existen ficheros EXCEL preparados por la OMS (disponibles en el sitio web de esta Organización) que facilitan los correspondientes cálculos, siempre que se tengan los "insumos" necesarios.

8. Cálculo de la carga por cada enfermedad en 2 atribuible a los relevantes factores de riesgo en 1, usando las fórmulas [3] y [4].

Los tres insumos fundamentales para el cálculo de la carga de los factores de riesgo son:

- la carga (negativa) de las enfermedades, expresada en términos de mortalidad, YLL, YLD, o DALYS. 
- el grado o nivel de exposición de la población a los factores de riesgo de interés.

- el Riesgo Relativo (RR) que vincula cada factor de riesgo con las correspondientes enfermedades.

A partir de esta información, y empleando las expresiones [3] y [4], se obtiene la carga atribuible a cada uno de los factores de riesgo; la carga que se distribuye puede ser la carga total (la generada por todas las enfermedades) o determinadas subcargas (las generadas por determinados subgrupos de enfermedades).

9. Análisis de la confiabilidad de los resultados obtenidos.

El análisis de la confiabilidad en los estudios de Carga de Factores de Riesgo se ha dividido en dos partes: la confiabilidad asociada a los modelos, y la confiabilidad asociada a los parámetros.

La confiabilidad asociada a los modelos se refiere a la incertidumbre existente en las relaciones que se establecen entre determinados factores de riesgo y determinadas enfermedades. Estas relaciones, en primer lugar, pueden o no ser ciertas; y en segundo lugar, el carácter de la relación puede ser muy diverso (relación lineal, no lineal, directa o inversa, entre otras). Otra incertidumbre asociada a modelos se produce cuando datos para algunos subgrupos poblacionales (subgrupos determinados por un cierto territorio, sexo, y grupo de edad, por ejemplo), no están disponibles y es necesario extrapolar desde los datos existentes para otros subgrupos.

La confiabilidad asociada a los parámetros se refiere al hecho de que un número importante de datos usados en los estudios de Carga de Factores de Riesgo, por ejemplo el grado de exposición a los factores de riesgo en la población de interés, suelen obtenerse a partir de muestras aleatorias más o menos representativas. EI error de muestreo implícito en estas estimaciones afecta también la confiabilidad de las estimaciones de las cargas atribuibles.

Lo menos que debe hacerse en un estudio de carga de factores de riesgo es reportar lo más profusamente posible las incertidumbres de estos dos tipos, de manera que los usuarios de los resultados puedan aquilatar el margen de error probable que tienen las estimaciones de las cargas atribuibles.

Otros procedimientos más sofisticados para cuantificar estas incertidumbres utilizan métodos de Monte Carlo y programas de computación al efecto, por ejemplo el @RISK. ${ }^{7}$

\section{LA CARGA DE FACTORES DE RI ESGO EN CUBA. ALGUNOS COMENTARI OS Y SUGERENCI AS ACERCA DE SU I MPLEMENTACI ÓN}

En esta sección se haran algunas reflexiones acerca de cómo se debieran implementar, adaptar y/o ajustar los procederes generales descritos en la sección anterior, al caso cubano. Estas reflexiones se harán apoyándose en los mismos nueve pasos presentados y discutidos en la sección anterior. 
Pasos 1 y 2: "Elección de los factores de riesgo", y "Elección de las enfermedades y lesiones relevantes que tienen como causa cada uno de los factores de riesgo en 1."

Tomando en cuenta la cantidad y calidad de la información obtenida por la OMS en sus estudios de Carga de Factores de Riesgo relacionada con las estimaciones de los riesgos relativos entre factores de riesgo y enfermedades específicas, ${ }^{4-6}$ parece adecuado, como una de varias alternativas, utilizar en los estudios cubanos los factores de riesgo y las enfermedades empleadas por la OMS.

Otras alternativas usarán factores y/ o enfermedades diferentes (a partir de las realidades e intereses proios, información endógena proveniente de expertos cubanos, y otros), y siempre que se cuente con las propias estimaciones de los correspondientes riesgos relativos.

La alternativa de usar los factores de riesgo y las enfermedades que usa la OMS, tiene como atractivo que permitiría comparar los resultados obtenidos con los de otras regiones del mundo.

Paso 3: "Elección de la variable de exposición adecuada para cada factor de riesgo seleccionado en el paso 1."

Las estimaciones de la OMS para los riesgos relativos entre factores de riesgo y enfermedades se hicieron para determinadas variables de exposición asociadas a los factores de riesgo estudiados. ${ }^{4,6}$ Usar otras variables de exposición (es decir, otras "operacionalizaciones" de esos factores de riesgo) puede hacerse siempre que se tengan las propias estimaciones para los correspondientes riesgos relativos.

En Cuba la principal fuente de información para obtener las distribuciones de exposición a los factores de riesgo más importantes es la "Encuesta Nacional de Factores de Riesgo", la que se reitera periódicamente.

Sería conveniente que la "operacionalización" de estos factores de riesgo en esas encuestas permita establecer una equivalencia con las variables de exposición empleadas por la OMS en sus estudios. ${ }^{4,6}$

Paso 4: "Obtención de información acerca de la distribución de exposición en la población a cada uno de los factores de riesgo en el paso $1 . "$

En el paso 3 ya se mencionó la conveniencia de que la "Encuesta Nacional de Factores de Riesgo" permitiera adaptar sus resultados a las variables de exposición empleadas por la OMS. De ser esto posible, la distribución de exposición a los factores de riesgo obtenida de estas encuestas podría ensamblarse "fisiológicamente" con las estimaciones de riesgos relativos obtenidas por la OMS.

Como ya se mencionó, otras alternativas de factores de riesgo ( $\mathrm{y} / \mathrm{o}$ variables de exposición) podrán y deberán (eventualmente) considerarse a partir de las realidades e intereses propios, siempre que vayan acompañadas de las propias estimaciones de los correspondientes riesgos relativos.

Es conveniente, por último, que los resultados de la "Encuesta Nacional de Factores de Riesgo" permita desglosar las prevalencias de exposición según sexo y grupos de edad; los grupos de edad empleados por la OMS se presentan en el paso 4 de la sección anterior. Si además, los resultados se desglosan por provincias, mucho mejor. 
Pasos 5 y 6: "Especificación de la relación (riesgo relativo) entre cada enfermedad en 2 y los relevantes factores de riesgo en el paso 1" y "Elección de la distribución de exposición hipotética de referencia (o escenario alternativo de referencia) para cada uno de los factores de riesgo en el paso 1."

Como ya se expresó, las estimaciones de riesgos relativos para los factores y enfermedades empleados por la OMS fueron estimados por ellos, a partir de revisiones exhaustivas de la evidencia científica disponible. No se harían las estimaciones propias si se usan los mismos factores y enfermedades que la OMS (tal y como se argumenta en los Pasos 1 y 2 de esta sección).

Las distribuciones alternativas de referencia (distribuciones mínimo-teóricas) también han sido postuladas por la OMS. ${ }^{4-6}$ Se propone usarlas en una primera aproximación.

Paso 7: "Cálculo de la carga (YLL, YLD, DALYs, y otros) para cada enfermedad en el paso 2."

La mortalidad puede obtenerse de los registros de mortalidad de la Dirección Nacional de Estadísticas (DNE) del MINSAP; los YLL (Años de Vida Potencial Perdidos por Muertes Prematuras, AVPP por muertes prematuras) se calculan también a partir de la mortalidad (desglosada por causa básica, sexo y edad). Para obtener los YLD y los DALYs se requieren registros de morbilidad, más difíciles de encontrar y en todos los casos menos confiables.

Los autores de este trabajo, junto con otros colegas, han estimado la carga de las enfermedades por muertes prematuras (YLL, o AVPP por muertes prematuras), en Cuba usando fundamentalmente un listado de causas (enfermedades y lesiones) ajustado al contexto cubano. ${ }^{9}$ Recalcular la carga usando el listado de enfermedades y lesiones de la OMS (listado que agrupado se convierte en el que se presenta en el recuadro 2) no constituye una dificultad importante. Luego este tema podría quedar resuelto por los especialistas cubanos.

Paso 8: "Cálculo de la carga por cada enfermedad en 2 atribuible a los relevantes factores de riesgo en 1, usando las fórmulas [3] y [4]". Apoyándose en los resultados y procedimientos que la $\mathrm{OMS}^{4,7}$ ha puesto al alcance de la comunidad científica internacional, los autores de este trabajo y otros colegas podrían calcular las fracciones atribuibles para las 240 combinaciones resultantes de combinar sexo ( 2 categorías), edad ( 8 categorías), y provincia (si se incluyen, 15 categorías), para cada pareja factor de riesgo-enfermedad que se incluya en el estudio.

Paso 9: "Análisis de la confiabilidad de los resultados obtenidos."

Parte de la ( falta de) confiabilidad en los resultados obtenidos se asocia a las imprecisiones en las estimaciones de los factores de riesgo que ha hecho la OMS para todas las combinaciones factores de riesgo-enfermedades estudiadas. ${ }^{4-6}$ Estas incertidumbres están documentadas y pueden ser reportadas.

La otra incertidumbre importante proviene de las imprecisiones en las estimaciones que haga la "Encuesta Nacional de Factores de Riesgo" del nivel de exposición a los distintos factores de riesgo estudiados. Los responsables de esta Encuesta deberán aportar información relevante en este sentido.

De todas maneras, y sin despreciar el valor de este paso 9, la peor estimación de la carga de los factores de riesgo es la que no se hace. Luego primero hay que 
ocuparse de hacer una estimación con el mayor rigor posible; después ya habrá tiempo de documentar el margen de error en las estimaciones realizadas.

\section{REFERENCI AS BI BLI OGRÁFI CAS}

1. Ezzati M, Lopez AD, Rodgers A, Murray CJ L, editors. Comparative quantification of health risks global and regional burden of disease attributable to selected major risk factors. Vol.1,2. Geneva:WHO; 2004.

2. Lopez AD, Mathers CD, Ezzati M, Jamison DT, Murray CJ L, editors. Global Burden of Disease and Risk Factors. Londres: Oxford University Press and World Bank $(\mathrm{s} / \mathrm{a})$.

3. Murray CJ L, Ezzati M, Lopez AD, Rodgers A, Vander Hoorn S. Comparative quantification of health risks: conceptual framework and methodological issues. Population Health Metrics. 2003; 1(1): 1.

4. Murray $C J L$, Lopez $A D$, editors. The global burden of disease: a comprehensive assessment of mortality and disability from diseases, injuries and risk factors in 1990 and projected to 2020. Cambridge, M.A: WHO; 1996. [Global Burden of Disease and Injury, Vol. 1. Harvard School of Public Health on behalf of WHO].

5. Murray CJ L, Lopez AD. Global mortality, disability, and the contribution of risk factors: global burden of disease study. Lancet. 1997; 349:1436-42.

6. Murray $\mathrm{CJ}$ L, Lopez $A D$. On the comparable quantification of health risks: lessons from the global burden of disease. Epidemiology. 1997; 10:594-605.

7. Mathers CD, Vos T, Lopez AD, Salomon J, Ezzati M, editors. National Burden of Disease Studies: A Practical Guide. Edition 2.0. Global Program on Evidence for Health Policy. Geneva: WHO; 2001.

8. Bradford Hill A. The Environment and disease: Association or Causation? Proceedings Royal Society Med. 1965;58:295-300.

9. Seuc AH, Dominguez E, Gallardo U, Garcia RM, Lopez L, González-Ochoa E. Mortalidad y Años de Vida Potencial Perdidos por muertes prematuras en mujeres cubanas: 1990, 1995 y 2000. Rev Cubana Salud Pública. 2004; 30(4).

Recibido: 7 de noviembre de 2007.

Aprobado: 2 de julio de 2008. 
Armando H. Seuc. Instituto de Angiología y Cirugía Vascular. La Habana, Cuba. E-mail: metodoli@infomed.sld.cu 